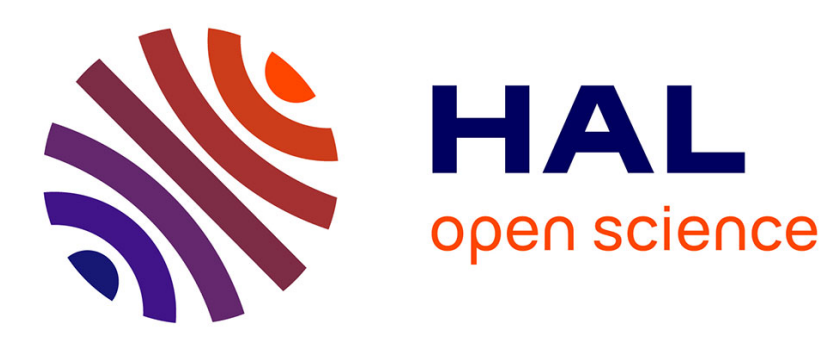

\title{
A fluctuation-corrected functional of convex Poisson-Boltzmann theory
}

R. Blossey, A C Maggs

\section{To cite this version:}

R. Blossey, A C Maggs. A fluctuation-corrected functional of convex Poisson-Boltzmann theory. Journal of Physics A: Mathematical and Theoretical, 2018, 51 (38), pp.385001. 10.1088/17518121/aad352 . hal-02344717

\section{HAL Id: hal-02344717 \\ https://hal.science/hal-02344717}

Submitted on 4 Nov 2019

HAL is a multi-disciplinary open access archive for the deposit and dissemination of scientific research documents, whether they are published or not. The documents may come from teaching and research institutions in France or abroad, or from public or private research centers.
L'archive ouverte pluridisciplinaire HAL, est destinée au dépôt et à la diffusion de documents scientifiques de niveau recherche, publiés ou non, émanant des établissements d'enseignement et de recherche français ou étrangers, des laboratoires publics ou privés. 


\title{
A fluctuation-corrected functional of convex Poisson-Boltzmann theory
}

\author{
R Blossey ${ }^{1}$, A C Maggs ${ }^{2}$ \\ ${ }^{1}$ University of Lille, Institute of Structural and Functional Glycobiology, CNRS \\ UMR8576, 59000 Lille, France \\ 2 CNRS UMR7083, ESPCI Paris, PSL Research University, 10 rue Vauquelin, Paris, \\ 75005 , France
}

\begin{abstract}
Poisson-Boltzmann theory allows to study soft matter and biophysical systems involving point-like charges of low valencies. The inclusion of fluctuation corrections beyond the mean-field approach typically requires the application of loop expansions around a mean-field solution for the electrostatic potential $\phi(\mathbf{r})$, or sophisticated variational approaches. Recently, Poisson-Boltzmann theory has been recast, via a Legendre transform, as a mean-field theory involving the dielectric displacement field $\mathbf{D}(\mathbf{r})$. In this paper we consider the path integral formulation of this dual theory. Exploiting the transformation between $\phi$ and $\mathbf{D}$, we formulate a dual Sine-Gordon field theory in terms of the displacement field and provide a strategy for precise numerical computations of free energies beyond the leading order.
\end{abstract}

\section{Introduction}

The description of electrolytes in soft matter is commonly based on the PoissonBoltzmann equation, a mean-field theory for the electrostatic potential $\phi(\mathbf{r})$ (see, e.g., [1], and references therein). Formally, this theory is derived from the partition function of a Coulomb system by performing a Hubbard-Stratonovich transformation to an (imaginary) fluctuating field $\phi(\mathbf{r})$, later identified with the electrostatic potential [2, 3]. (For the Hubbard-Stratonovich transformation, see e.g. [4, 5, 6, 7, 8, 9]). This approach is valid in the weak fluctuation regime, in which the saddle-point of the action yields the standard Poisson-Boltzmann equation, which can be systematically improved by a loop expansion [3]. An alternative approach to go beyond mean-field theory is to invoke a variational approach [10, 11]. Going beyond mean field in Poisson-Boltzmann is known to improve many features of the theory, for instance dielectric contrast and image-charge interactions are much better described in theories which allow fluctuations [12].

A difficulty with these approaches is that the action of the functional integral is complex. A Wick rotation gives a real theory, but at the price of rendering the effective free energy concave. Thus, when the electrostatics is coupled to other degrees of freedom, the extremization of the free energy becomes a numerically difficult operation. Extrema result from a combination of minima in the non-electrostatic degrees of freedom and maxima in the electrostatic potential. This complicates many 
numerical studies of biophysical molecules in which the solvent is described by PoissonBoltzmann theory, and also has repercussions for the validity limits of theories including fluctuation effects beyond Poisson-Boltzmann theory [13]. This technical problem has been circumvented [14, 15] by a reformulation of the Poisson-Boltzmann theory in terms of purely convex functionals, which is achieved by the means of a Legendre transform, with the complex field $\phi(\mathbf{r})$ being systematically replaced by the dielectric displacement field $\mathbf{D}(\mathbf{r})$. Expressed in terms of the field $\mathbf{D}(\mathbf{r})$, the resulting theory yields a convex functional so that standard minimization techniques can be applied, and loop corrections or variational approaches be defined as for the original Poisson-Boltzmann theory. Though it was constructed only from the mean-field, the one-loop correction in the dual theory (very surprisingly) has been shown to yield the same fluctuation spectrum as obtained within the usual formulation of Poisson-Boltzmann theory [16].

This approach, however, working from conventional mean-field theory, does not help in constructing a systematic improvement in the dual formulation of the theory. The aim of the present article is to formulate a systematic method for going beyond the "oneshot" approximation that has been considered until now for constructing dual theories and present a general formulation that is equivalent to the exact, discretized statistical mechanics of the electrolytes that are formulated as integrals over an electrostatic potential. We stress the generality of our approach, even if in this paper we chose to apply the formulation to only the simplest two-component electrolyte.

With this motivation in mind, we revisit the dual approach from a functional integral perspective which relies on the introduction of the dual transform via delta functions. The arising transformation integrals can be performed either in an exact way, or in a systematic fashion by invoking the Poisson summation formula. The latter will first give us access to a saddle-point like limit, but it equally allows us - with no more than simple integration and one-dimensional Fourier transforms - to explicitly calculate the corrections beyond the saddle point which arise from the discrete nature of the charges. In this way we obtain a novel formulation of Poisson-Boltzmann theory in terms of its dual which is exact. Our final result is a discretized free energy function, which, when sampled gives the exact discretized energy of a set of discrete charges interacting with the Coulomb interaction.

We expect that this novel dual formulation will be highly useful for the finite temperature simulation of coupled conformational/electrostatic degrees of freedom, rather than the minimization envisaged with the original mean-field theory. For this purpose we formulate the theory in a formulation adequate for numerical computations on lattices.

The paper is organized as follows. In the second section, we discuss the notion of duality, recall the formulation of the duality transform for the Poisson-Boltzmann functional and the derivation of the dual mean-field functional. In section three we derive a prescription to formulate the functional integral of a dual theory including fluctuations beyond mean field for the case of the symmetric electrolyte; this choice is made for definiteness and provides no restriction to the method. The program is 
subsequently implemented in section four. We conclude in section five.

\section{Duality in Poisson-Boltzmann theory}

We first recall the notion of duality previously employed in the context of the PoissonBoltzmann theory at the mean-field level [14]. We start with the usual expression [2] for the partition function for a set of charged particles interacting through the Coulomb interaction, described through a fluctuating potential field $\phi$ :

$$
Z=\int D[\phi] e^{-\beta \int d^{3} \mathbf{r} h(\phi)}
$$

with

$$
h(\phi)=\varepsilon \frac{(\nabla \phi)^{2}}{2}+g(i \phi)-i \varrho_{f} \phi,
$$

where $g(i \phi)$ is the pressure, while $\varrho_{f}$ is the density of fixed charges, which are typically confined to a small part of the system. Introducing the electric field $\mathbf{E}$ with the help of a delta function (and being free with non-essential normalization factors)

$$
Z=\int D[\phi] D[\mathbf{E}] e^{-\beta \int d^{3} \mathbf{r} h(\phi)} \delta(\mathbf{E}+\nabla \phi)
$$

yields after Fourier representation of the delta function with multiplier D

$$
Z=\int D[\phi] D[\mathbf{E}] D[\mathbf{D}] e^{-\beta \int d^{3} \mathbf{r} h(\phi, \mathbf{E}, \mathbf{D})}
$$

with

$$
\begin{aligned}
h(\phi, \mathbf{E}, \mathbf{D}) & =\varepsilon \frac{\mathbf{E}^{2}}{2}+g(i \phi)-i \mathbf{D} \cdot(\nabla \phi+\mathbf{E})-i \varrho_{f} \phi \\
& =\varepsilon \frac{\mathbf{E}^{2}}{2}+g(i \phi)-i \mathbf{D} \cdot \mathbf{E}+i \phi\left(\operatorname{div} \mathbf{D}-\varrho_{f}\right) .
\end{aligned}
$$

Careful consideration of boundary conditions shows that

$$
\oint \phi \mathbf{D} \cdot d \mathbf{S}=0
$$

so that one requires either $\phi=0$ or the normal component of the field $\mathbf{D}_{n}=0$ at the boundaries. Performing the integration over the electric field $\mathbf{E}$, one finds

$$
Z=\int D[\phi] D[\mathbf{D}] e^{-\beta \int d^{3} \mathbf{r} h(\phi, \mathbf{D})}
$$

with

$$
h(\phi, \mathbf{D})=\frac{\mathbf{D}^{2}}{2 \varepsilon}+g(i \phi)+i \phi\left(\operatorname{div} \mathbf{D}-\varrho_{f}\right) .
$$


In this expression, $\operatorname{div} \mathbf{D}-\varrho_{f} \equiv s$ can be read as a Fourier transform variable such that we arrive at the formal expression

$$
h(\phi, \mathbf{D})=\frac{\mathbf{D}^{2}}{2 \varepsilon}-\ln \left\{\mathcal{F}\left(e^{g(i \phi)}\right)\right\}\left[\operatorname{div} \mathbf{D}-\varrho_{f}\right]
$$

with $\mathcal{F}$ the Fourier operator. We emphasize that this step requires the evaluation of just a single one-dimensional Fourier transform. In numerical applications this transform can be done once and tabulated, even if the model for $g(i \phi)$ is relatively complicated analytically. As emphasized in [17] the function $g(\phi)$ is typically the pressure of the uncharged fluid. We then find the main result of this paper

$$
Z=\int D[\mathbf{D}] e^{-\beta \int d^{3} \mathbf{r} h_{e f f}(\mathbf{D})}
$$

in which $h_{\text {eff }}(\mathbf{D})$ is a local functional of $\mathbf{D}$. If the conventional saddle-point approximation is employed, we obtain the standard mean-field theory of soft matter electrostatics. Our aim here is to derive an expression for $h_{\text {eff }}(\mathbf{D})$ valid beyond meanfield theory, on the basis of which an exact sampling of the partition function eq.(4) can be performed e.g. by Monte-Carlo simulation [18].

In our formulation of the full statistical mechanics of the dual theory it is a crucial feature that the duality transformation $\phi \rightarrow \mathbf{D}$ has been carried out solely via the introduction of delta functions. Only this approach guarantees the identity of the fluctuation spectra of the dual theory as discussed in detail [19] in a general field-theory setting. By contrast, theories derived from reparametrizations are called pseudo-dual theories [19] for which this property in general does not hold. This is e.g. the case for the reparametrizations of the Poisson-Boltzmann theory recently developed in [20, 21] with the aim of defining convex functionals for the electrostatic potential. These theories fail to produce the correct fluctuation spectra as was shown explicitly in the calculation of the one-loop correction [16]. Even in lattice gauge theories, dual formulations can have similar technical advantages of positivity and convexity and are an active research topic [22].

The above presentation has been at a formal level. In order to be explicit in this program we will now present a detailed calculation of eq. (3) for the case of a simple model for the pressure,

$$
g(i \phi)=-2 \lambda \cos (\phi e \beta)
$$

corresponding to a perfect gas model of the ions. The full partition function corresponding to (5) (without the complex coupling to the external charge $\varrho_{f}$ ) is the well-known Sine-Gordon energy. In three dimensions it has been widely studied due to its links to electrodynamics. Unlike the closely related model of charged hard-spheres there is no phase transition [23]. In its discretized form the theory is regularized by the lattice spacing.

For both the purpose of regularizing and simulating this theory, we will first discretize it. Our dual model then is, by construction, entirely equivalent to this full, 
discrete Sine-Gordon system, as it includes all the fluctuations beyond mean-field theory that are contained in this starting theory.

\section{Discretization of the dual theory}

We now turn to a definition and calculation of the functional integral (2), for the explicit case of the symmetric electrolyte given by (5). The continuum action for the potential that we start with is

$$
h(\phi)=\varepsilon \frac{(\nabla \phi)^{2}}{2}-2 \lambda \cos (\phi e \beta)-i \varrho_{f} \phi .
$$

We discretize this model at a spacing $\ell$ to a simple cubic lattice. The potential and charges are associated with the sites of the lattice. Derivatives are replaced by lattice differences, $d_{\ell}$. The discretized energy corresponding to eq. (6) is then

$$
h_{\ell}(\phi)=\ell \varepsilon \frac{\left(d_{\ell} \phi\right)^{2}}{2}-2 \lambda \ell^{3} \cos (\phi e \beta)-i \ell^{3} \varrho_{f} \phi .
$$

We change the integration variable from $\phi$ to the scaled potential $\phi /(e \beta)$, which gives the (a-dimensional) statistical weight

$$
\beta h_{\ell}(\phi)=\sum_{\text {sites }}\left[\frac{\ell \varepsilon}{e^{2} \beta} \frac{\left(d_{\ell} \phi\right)^{2}}{2}-2 \bar{\lambda} \cos (\phi)-i \bar{\varrho} \phi\right],
$$

where we define $\bar{\lambda}=\beta \ell^{3} \lambda$ and $\bar{\rho}=\varrho_{f} \ell^{3} / e$. We note that $\ell \varepsilon / e^{2} \beta=\ell /\left(4 \pi \ell_{B}\right)$, with $\ell_{B}$ the Bjerrum length. To define the dual formulation we now consider the discrete electric field, $\mathbf{E}_{\ell}=-d_{\ell} \phi$, to be associated the links of the lattice and impose this constraint using a delta-function integral over $\mathbf{D}_{\ell}$, which is also considered as link variable. The entire formulation follows very closely the continuum calculation given above. We need only to define the discrete equivalent of the divergence operator from the adjoint (or matrix transpose) of the difference operator $d_{\ell}: \operatorname{div} \rightarrow-d_{\ell}^{T}[24]$.

The partition function in its discretized form is thus

$$
Z=\int D\left[\mathbf{D}_{\ell}\right] D[\phi] e^{-\sum\left(\frac{e^{2} \beta}{2 \ell \varepsilon} \mathbf{D}_{\ell}^{2}+i\left(-d_{\ell}^{T} \mathbf{D}_{\ell}-\bar{\varrho}\right) \phi-2 \bar{\lambda} \cos (\phi)\right)}
$$

This expression can be reorganized into

$$
\begin{aligned}
Z & =\int D\left[\mathbf{D}_{\ell}\right] e^{-\sum \frac{e^{2} \beta}{2 \ell \varepsilon} \mathbf{D}_{\ell}^{2}} \int D[\phi] e^{\sum(-i s \phi+2 \bar{\lambda} \cos (\phi))} \\
& =I\left[\mathbf{D}_{\ell}, J[\phi]\right]
\end{aligned}
$$

with $s \equiv-d_{\ell}^{T} \mathbf{D}_{\ell}-\bar{\varrho}$. Here sums in the exponentials are understood as being over sites for potentials and charges and over links for field components. It is here that we see explicitly that the only step that is needed to complete the program is the integration over the field $\phi$. As the field $\phi$ in the expression (7) is purely local, we can drop the 
functional notation and thus have to deal with a simple integral for each site of the discretization

$$
z(s)=\int d \phi e^{(-i s \phi+2 \bar{\lambda} \cos (\phi))} .
$$

For a general model of electrolyte we can expect that this integral will be difficult to perform analytically, but it can always be treated in a numerical manner, in order to generate the exact effective theory for $\mathbf{D}_{\ell}$. We can make further analytic progress for the case of the two-component electrolyte, which we now present in detail.

\section{Two-component electrolyte}

The standard way of proceeding to calculate $z(s)$ would be to invoke complex integration. The argument of the exponential function has zeroes at $2 \bar{\lambda} \sin (\phi)=i s$, hence at $\phi=\sin ^{-1}(i s / 2 \bar{\lambda})$, whereby the inversion of the sine function fixes the saddle to be retained. Here, we will work through the saddle-point evaluation and its corrections in a different manner, in view of our interest to have an approach which is adapted to numerical computations.

The mathematical tool we use in our formalism is the Poisson summation formula which expresses a sum over integer occupation numbers by an equivalent sum over Fourier coefficients. This gives a mathematically rigorous formulation in which the dominant contribution is the mean-field free energy already but gives a framework in which higher-order corrections to the dual theory are expressed as an exact Fourier series, with exponential convergence. Thus we now find an analytic expansion for eq. (8).

In order to implement this program we note that we first rewrite $z(s)$ in the discrete form

$$
z(s)=\sum_{n} \delta(s-n) g(n)=\sum_{n} \delta(s-n) e^{-f_{e}(n)}
$$

where we then need to calculate the weighting function $f_{e}(s)$. In order to perform this step we expand the exponentiated cosine in eq. (8) as a Taylor series of the two complex exponentials using

$$
\exp \left(\bar{\lambda} e^{i \phi}\right)=\sum_{n=0}^{\infty} \frac{e^{i n \phi+n \mu}}{n !},
$$

where for convenience we define $\mu=\ln (\bar{\lambda})$. Substituting eq. (10) in eq. (8) and using the definition of the delta function we find

$$
z(s)=\sum_{n_{1}, n_{2}=0}^{\infty} \frac{1}{n_{1} !} \frac{1}{n_{2} !} e^{\mu\left(n_{1}+n_{2}\right)} \delta\left(s+n_{1}-n_{2}\right)
$$

where $n_{1}$ and $n_{2}$ represent the occupation numbers of positive and negative ions. 
In order to perform the summation, we can invoke the Poisson summation formula which tells us that if $g(n, s)$ is a function defined on integers and $\tilde{g}$ is its continuous Fourier transform

$$
z(s)=\sum_{n} g(n, s)=\sum_{k} \tilde{g}(2 \pi k, s)=\sum_{k} z_{k}(s)
$$

where $z_{k}(s)$ is the contribution to the $k$-th Fourier mode to the total sum $z(s)$. In order to apply this identity we solve the delta-function constraint and reduce the problem to a single summation, which leads to the expression

$$
z(s)=\sum_{n} g(n, s)=\sum_{n} \frac{1}{n !} \frac{1}{(n+|s|) !} e^{\mu(2 n+|s|)} .
$$

This expression is a simple function of the variable $s$, which can be tabulated, or interpolated to arbitrary precision for numerical work.

Some additional care is, however, needed for our problem: we here require the sum is over positive integers, whereas the Poisson formula applies for a sum over positive and negative integers. This can be achieved by imposing a multiplicative weighting function.

We implement this first for the $k=0$ contribution to the full partition sum eq. (12). For convenience we go back to a continuous variable $n$ with which we can write this contribution as an integral over the summand of eq. (12); it thus is the naive integral over the occupation number variable, forgetting about the discrete nature of the elementary charges. It reads as

$$
z_{0}(s)=\int_{-\infty}^{\infty} \mathrm{d} n w(n) g(n, s)
$$

where the weighting function is given by, e.g.,

$$
w(n)= \begin{cases}0 & \text { if } n<-1 \\ \frac{e^{-1 / n}}{e^{-1 / n}+e^{1 /(1+n)}} & \text { if }-1<n<0 \\ 1 & \text { if } n>0 .\end{cases}
$$

This cross-over function is smooth and increases from zero to unity on the interval $-1<n<0$. The choice of $w(n)$ is non-unique; we note that the general theory of Fourier analysis show that the final results does not depend on the particular choice we have made. We also note that the extension of the factorial function to the real numbers, which is needed to represent $g(n, s)$ in its continuous form, is also non-unique. The commonly used extension is Euler's Gamma function, but alternative extensions due to Hadamard are also possible [25].

The integral $z_{0}(s)$ is dominated for $\bar{\lambda}>1$ by a saddle. We will also show that in this limit the contributions $z_{k}(s)$ for $|k| \geq 1$ are exponentially smaller and that to high accuracy $z(s)$ can be replaced by $z_{0}(s)$. To study the nature of the saddle we work with an improved version of the Stirling formula

$$
\ln (n !) \approx(n+1 / 2) \ln (n+1 / 2)-(n+1 / 2)+O(1)=S(n)
$$


which correctly includes the term $(\ln n) / 2$ in its expansion. Replacing the single continuous variable $n$ again by two continuous variables $n_{1}$ and $n_{2}$ we then have the expression

$$
z_{0}(s)=\int_{n_{1}, n_{2}} \exp \left(\mu\left(n_{1}+n_{2}\right)-S\left(n_{1}\right)-S\left(n_{2}\right)\right) \delta\left(s+n_{1}-n_{2}\right)
$$

The saddle-point is best studied by implementing the delta-function constraint with a Lagrange multiplier $\tau$, which leads to the following equations,

$$
\begin{aligned}
s & =2 \bar{\lambda} \sinh (\tau) \\
n_{1}+1 / 2 & =\bar{\lambda} e^{-\tau} \\
n_{2}+1 / 2 & =\bar{\lambda} e^{+\tau} .
\end{aligned}
$$

On eliminating $\tau$ we find the values of the occupation numbers which extremize the integrand:

$$
\begin{aligned}
& n_{2}+1 / 2=\frac{\left(s+\left(s^{2}+4 \bar{\lambda}^{2}\right)^{1 / 2}\right)}{2} \\
& n_{1}+1 / 2=\frac{\left(-s+\left(s^{2}+4 \bar{\lambda}^{2}\right)^{1 / 2}\right)}{2} .
\end{aligned}
$$

When $(s / \bar{\lambda})$ is small, both $n_{1}$ and $n_{2}$ equal the background occupation number $\bar{\lambda}$. The value of the saddle-point eq. (13) is

$$
\begin{array}{r}
\ln \left(z_{0}\right) \approx s \ln \left[\frac{s}{2 \bar{\lambda}}+\sqrt{1+(s / 2 \bar{\lambda})^{2}}\right] \\
-\sqrt{s^{2}+4 \bar{\lambda}^{2}}-\ln (\bar{\lambda})
\end{array}
$$

and we define $f_{0}(s)=-\ln z_{0}$. With $s$ small, $z_{0}=O\left(e^{2 \bar{\lambda}}\right)$.

We now evaluate the second derivative, $\Delta(s)$, of the action at the saddle

$$
\Delta(s)=\frac{1}{n_{1}+1 / 2}+\frac{1}{n_{2}+1 / 2}=\frac{1}{\bar{\lambda}^{2}} \sqrt{4 \bar{\lambda}^{2}+s^{2}} .
$$

For small $s, \Delta(s)=2 / \bar{\lambda}$. At the saddle-point we have the quadratic approximation to the statistical weight,

$$
f(n, s)=f_{0}(s)+\frac{1}{2} \Delta\left(n-n_{s}\right)^{2}, \quad n_{s}=\min \left(n_{1}, n_{2}\right) .
$$

According to the sign of $s$ we use the smaller of $n_{1}$ and $n_{2}$ as the primary variable mean-field occupation number. Performing the integral over the full occupation number $n$ in eq. (17) we find that the effective action for eq. (9) is

$$
f_{e}(s)=f_{0}(s)+\frac{1}{2} \ln (\Delta(s)) .
$$



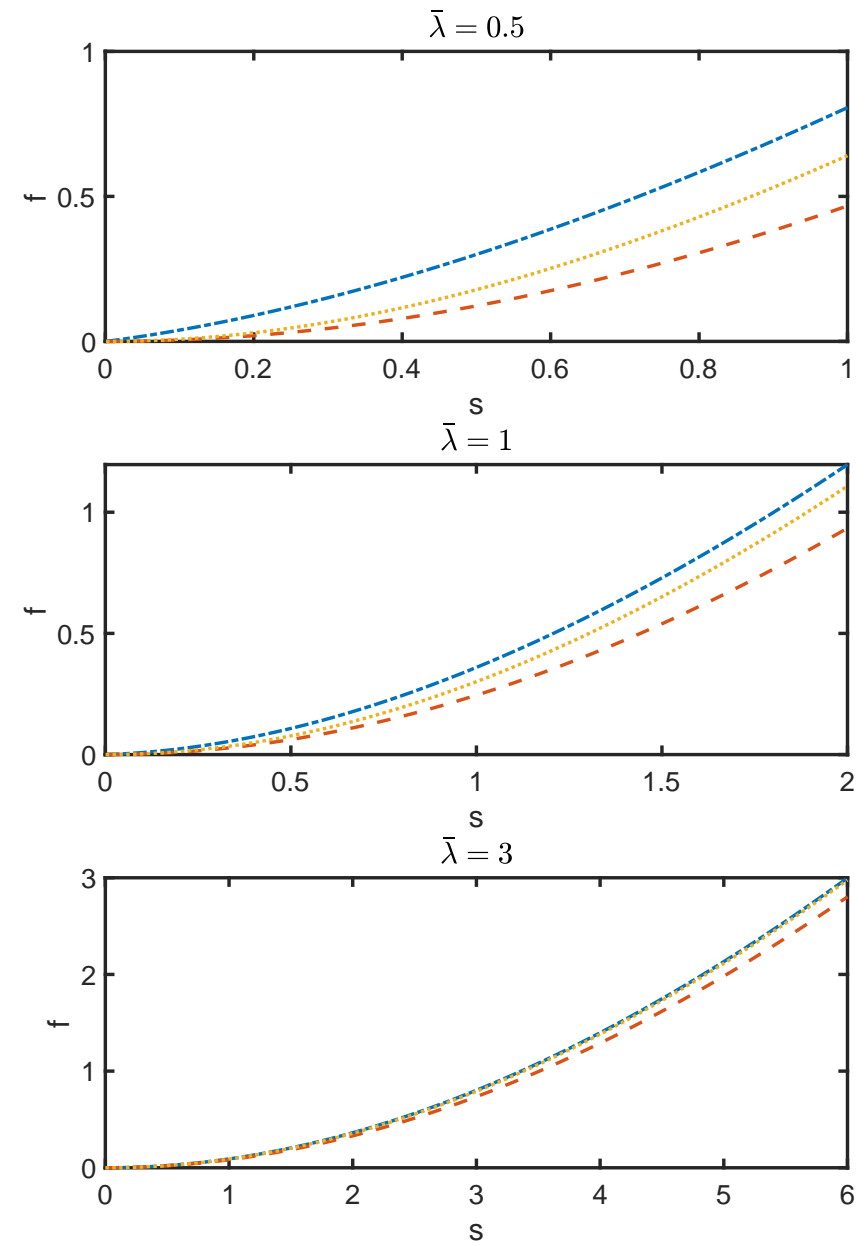

Figure 1. Comparison of the evaluation of the sum eq. 111), (blue dot-dash) compared to the saddle point approximation eq. (16), (red dash) and the quadratic approximation, eq. (18), (yellow dotted). Three different values of $\bar{\lambda}$. For already modest values of $\bar{\lambda}=3$ (bottom) the quadratic approximation approximation is already excellent with a good overlay of the exact and approximate curves. For $\bar{\lambda}<1$ (top) the mean field and quadratic approximations underestimate the true free energy. The curves are shifted so that $f(0)=0$.

With the help of the Poisson summation formula we now calculate the higher Fourier contributions to the sum eq. (12) which come from the discrete nature of the elementary charges. For $\bar{\lambda}>1$ the Fourier components are also calculated in the saddlepoint approximation. We take the quadratic approximation to the energy eq. (17) and regroup the positive and negative Fourier coefficients,

$$
\tilde{z}_{k}=\left(z_{k}+z_{-k}\right)=e^{-f_{0}(s)} \int 2 \cos (2 \pi k n) e^{-\Delta\left(n-n_{s}\right)^{2} / 2} \mathrm{~d} n,
$$

which results in the expression

$$
\tilde{z}_{k}=\frac{e^{-f_{0}(s)}}{\sqrt{\Delta}} 2 \cos \left(2 \pi k n_{s}\right) e^{-2 k^{2} \pi^{2} / \Delta} .
$$


If we consider that $\Delta=O(2 / \bar{\lambda})$ then the amplitude of this contribution is

$$
\tilde{z}_{k} / z_{0}=O\left(e^{-\pi^{2} k^{2} \bar{\lambda}}\right) .
$$

If $\bar{\lambda} \gtrsim 1$ then this contribution is strongly suppressed in comparison with the $k=0$ contributions to the partition function.

We now calculate the importance of the crossover function $w(n)$ which constrains the sums and integrals to be over positive occupations. Near $n=0$ the function $\ln (n !)=O(1)$. The Fourier transform of the weighting function can be estimated by a further saddle-point calculation to be $O(1) \times e^{-\sqrt{k}}$, [26]. We thus expect that the contribution to the partition sum coming from the constraint of the sum over positive occupation numbers is of $O(1)$.

Finally, by a regrouping of the contributions we conclude that an expansion for the Fourier partition sum eq. 12 is given by the formula

$$
z(s)=\frac{e^{-f_{0}(s)}}{\sqrt{\Delta(s)}}\left(1+2 \cos \left(2 n_{s}\right) e^{-2 \pi^{2} / \Delta(s)}\right)+O(1),
$$

where $-f_{0}(s) \sim 2 \bar{\lambda}$ and we have kept just the first and largest non-trivial Fourier component for $\tilde{z}_{k}$ 团

We conclude that when the discretization is such that $\bar{\lambda}>1$, we can drop the oscillating terms as well as the end-point corrections to find the simplified expression

$$
f_{e}(s)=f_{0}(s)+\frac{1}{4} \ln \left(\frac{s^{2}+4 \bar{\lambda}^{2}}{\bar{\lambda}^{4}}\right) .
$$

This result is to be compared to the exact sum eq. (11) in Fig. 1. We see that the the analytic expression is an excellent fit to the full numerical evaluation.

Thus for $\bar{\lambda}>1$, the final effective functional for the displacement field $\mathbf{D}_{\ell}$ is given by the

$$
h_{e f f}=\sum_{\text {links }}\left[\frac{e^{2} \beta}{\ell \varepsilon} \frac{\mathbf{D}_{\ell}^{2}}{2}+f_{e}\left(-d_{\ell}^{T} \mathbf{D}_{\ell}-\bar{\varrho}\right)\right],
$$

where $f_{e}(s)$ is given by eq. (16) and eq. (19), and the measure includes the delta-functions of eq. (9). This functional is valid beyond mean-field as it includes the fluctuations in the underlying field $\phi$, it is also explicitly an energy function that should be sampled by Monte Carlo or molecular dynamics.

\section{Conclusions}

In this Letter we have given an explicit formulation of the duality-transformed PoissonBoltzmann theory, for the illustrative case of a system of a symmetric electrolyte. The

$\ddagger$ We have also assumed that the background charge density is not so large that it overwhelms the electrolyte concentration. 
partition function is given by a double integral over the potential-field variable $\phi$ and the dielectric displacement field $\mathbf{D}_{\ell}$. The potential integral can be evaluated as a systematic series with the help of the Poisson summation formula. The result is a Fourier series for the $\phi$ integral. For $\bar{\lambda}>1$ we evaluated this series analytically, but the Fourier transforms

can also be evaluated numerically for small $\bar{\lambda}$. The final result is a theory which has physical content identical to the Sine-Gordon model of a charged lattice gas, which goes beyond mean field theory and includes all fluctuations in an exact manner. The final theory remains clearly analytically intractable. However, it is of now of a form which is simple to simulate, and to couple to external charges. This is in contrast to the original field-theory formulation in terms of the potential, which included a complex coupling to external charges. This opens the possibility of simulation of complex coupled situations where electrostatic fluctuations are important.

Although we have derived our formulation for the case of the symmetric electrolyte our approach is not limited to this choice and should allow the treatment of more complex models of electrolyte, fully including fluctuations beyond mean-field theory in their coupling to external soft-matter systems.

\section{References}

[1] Naji A, Kandu M, Forsman J and Podgornik R 2013 The Journal of Chemical Physics 139150901

[2] Netz R and Orland H 2000 The European Physical Journal E 1 203-214

[3] Netz R 2001 The European Physical Journal E 5 557-574

[4] Samuel S 1978 Physical Review D 181916

[5] Kholodenko A and Beyerlein A 1986 Physical Review A 343309

[6] Caillol J and Raimbault L 2001 Journal of Statistical Physics 103 753-776

[7] Raimbault L and Caillol J 2001 Journal of Statistical Physics 103 777-799

[8] Caillol J 2004 Journal of Statistical Physics 115 1461-1504

[9] Tellez G 2004 Physical Review E $\mathbf{7 0} 01158$

[10] Netz R R and Orland H 2003 The European Physical Journal E 11 301-311

[11] Buyukdagli S and Blossey R 2016 Journal of Physics: Condensed Matter 28343001

[12] Xu Z and Maggs A 2014 Journal of Computational Physics 275310 - 322 ISSN 0021-9991

[13] Frydel D 2015 European Journal of Physics 36065050

[14] Maggs A C 2012 EPL (Europhysics Letters) 9816012

[15] Maggs A C 2002 The Journal of Chemical Physics 117 1975-1981

[16] Pujos J S and Maggs A C 2015 Journal of Chemical Theory and Computation 11 1419-1427

[17] Maggs A C and Podgornik R 2016 Soft Matter 12(4) 1219-1229

[18] Duane S, Kennedy A, Pendleton B J and Roweth D 1987 Physics Letters B 195216 - 222

[19] Fradkin E and Tseytlin A 1985 Annals of Physics $16231-48$

[20] Jadhao V, Solis F J and de la Cruz M O 2013 Phys. Rev. E 88(2) 022305

[21] Solis F J, Jadhao V and Olvera de la Cruz M 2013 Phys. Rev. E 88(5) 053306

[22] Gattringer C 2014 PoS LATTICE 2013002

[23] Kosterlitz J M 1977 Journal of Physics C: Solid State Physics 103753

[24] Lipnikov K, Manzini G and Shashkov M 2014 Journal of Computational Physics 2571163 - 1227

[25] Alzer H 2009 Abhandlungen aus dem Mathematischen Seminar der Universität Hamburg 79 11-23

[26] Johnson S G 2015 ArXiv e-prints (Preprint 1508.04376) 